\title{
Competencias digitales en estudiantes y docentes universitarios del área de la educación física y el deporte \\ Digital competences in university students and teachers in the area of Physical Education and Sports
}

\author{
Ricardo Leonardo Perea Rodríguez, Claudia Marcela Abello Avila \\ Universidad Manuela Beltrán (Colombia), Corporación Universitaria Minuto de Dios (Colombia)
}

\begin{abstract}
Resumen. Hace apenas unas décadas, el uso de herramientas digitales en la enseñanza de la educación física y el deporte era un asunto de escaso interés. No obstante, la acelerada incursión de las Tecnologías de la Información y la Comunicación (TIC) en la sociedad actual, ha venido convirtiendo en una necesidad imperiosa la adquisición de las denominadas competencias digitales. Este estudio se realizó con la participación de cuatro programas profesionales del ámbito de la educación física y el deporte de la ciudad de Bogotá, con el objetivo de determinar si existen diferencias significativas entre la percepción de estudiantes y docentes sobre el desarrollo de sus competencias digitales en dependencia de variables como la modalidad de estudio, el enfoque académico, el género, la edad, y para el caso de los docentes, también la experiencia profesional y su formación académica. A partir de los resultados hallados a través de las pruebas no paramétricas de KruskalWallis, con un nivel de significancia de .05, se determinó que tanto estudiantes como docentes perciben el desarrollo de sus habilidades TIC en un nivel medio alto y se desestimaron diferencias con relación al género y la edad. De otra parte, se concluyó que los estudiantes que hacen parte de programas de formación a distancia, y de línea pedagógica tienen una mejor percepción de estas habilidades a la vez que aquellos docentes con más experiencia y un nivel de formación superior.
\end{abstract}

Palabras claves: competencia digital, educación física, estudiantes, docentes, deporte.

\begin{abstract}
Just a few decades ago, the use of digital tools in the teaching of Physical Education and Sports was a matter of little interest. However, the expedited incursion of information and communication technologies (ICT) in today's society has made the acquisition of the so-called digital skills an imperative. This study was carried out with the cooperation of four professional programs in the field of Physical Education and Sports in Bogota, with the aim of determining whether there are significant differences between the perception of students and teachers about the development of their digital skills in dependence on variables such as study modality, academic approach, gender, age, and in the case of teachers, also professional experience and academic training. From the results found through the Kruskall Wallis non-parametric tests, with a significance level of .05, it was determined that both students and teachers perceive the development of their ICT skills at a medium-high level, and differences in relation to gender and age were ruled out. On the other hand, it was concluded that students who are part of distance learning programs, and from pedagogical line, have a better perception of these skills, as well as those teachers with more experience and a higher level of training.
\end{abstract}

Keywords: digital competence; physical education, students, Teachers, Sports.

\section{Introducción}

El desarrollo vertiginoso de la tecnología en la historia reciente de la humanidad ha supuesto importantes cambios en la configuración de las estructuras sociales actuales, llegando a constituirse como uno de sus atributos distintivos. La educación como esfera subyacente y fundamental de ésta, debe responder y adaptarse a los cambios derivados de la inevitable inmersión tecnológica y posibilitar su uso consciente, reflexivo y dinámico (Cela-Ranilla, Esteve, Esteve, González, \& Gisbert-Cervera, 2017; Guzmán-Simón, García-

Fecha recepción: 10-12-20. Fecha de aceptación: 21-09-21 Ricardo Leonardo Perea Rodríguez richyper1@hotmail.com
Jiménez, \& López-Cobo, 2017).

En este escenario, ya no será suficiente una formación basada en los conocimientos básicos de la lectura y la escritura, sino que se requerirá la adquisición de nuevas técnicas para el entendimiento y la interacción con las tecnologías (Fernández, Ordóñez, Morales, \& López, 2019). Las competencias digitales son el conjunto de habilidades, conocimientos y destrezas necesarias para responder a las exigencias propias del entorno pedagógico actual (Cabero-Almenara \& Palacios-Rodríguez, 2019; Rodríguez-García, Raso, \& Ruiz-Palmero, 2019). En estricto sentido, las competencias digitales hacen referencia a la movilización de habilidades y destrezas que permiten buscar, seleccionar críticamente, obtener y procesar información relevante a partir del uso 
de las TIC para transformarlas en conocimiento (Flores-Lueg \& Roig, 2016), razón por la cual existen numerosos estudios que pretenden constatar el dominio de estos saberes en estudiantes y docentes universitarios (Cabero-Almenara \& Palacios-Rodríguez, 2019; González-Zamar, Abad-Segura, \& Belmonte-Ureña, 2020; López-Gil \& Sevillano, 2020; Marcano, Íñigo, \& Sánchez, 2020).

Como señalan Llorente y Cabero (2010), el desarrollo de estas habilidades se perfila en la actualidad, como elemento primordial para la formación de los estudiantes universitarios, los cuales deben ser competentes en el dominio de unos códigos específicos, sistemas simbólicos y formas de interaccionar con las TIC.

Por su parte, el docente contemporáneo, como agente dinamizador del proceso educativo moderno debe ser capaz de avizorar más allá de las cualidades o características intrínsecas de las TIC y de su amplia capacidad de integrarse a los planteamientos didácticos como recurso de apoyo, y reconocer que su valor pedagógico se transforma en dependencia del contexto metodológico en el que sean empleadas (Batane \& Ngwako, 2017; Domingo-Coscollola, Onsès-Segarra, \& Sancho-Gil, 2018).

En tal sentido, el docente actual, debe estar en capacidad de analizar y valorar las transformaciones que las TIC han aportado al medio pedagógico, introduciendo innovaciones y procesos de mejora en los sistemas educativos, nuevas modalidades de formación, y el desarrollo de competencias digitales que permitan transformar el rol del estudiante y el suyo propio (Cabrera, 2020).

A pesar de su carácter fundamentalmente práctico, el área de la actividad física y el deporte no es ajena a estas dinámicas, diferentes estudios así lo constatan (Araújo, Batista, \& Moura, 2017; Merino-Campos \& del Castillo, 2016; Quijada, 2015), resaltando la importancia de la integración de las TIC en el proceso de formación en este ámbito y los requerimientos de estudiantes y docentes acorde a las dinámicas sociales actuales.

El objetivo general de esta investigación es analizar la autopercepción de estudiantes y docentes de cuatro programas profesionales del área de la actividad física y el deporte, sobre sus competencias digitales, y establecer la existencia de diferencias significativas a partir de variables tales como la modalidad de estudio, el enfoque de formación, el género, la edad, la experiencia y la formación. El reconocer en qué grado responden las competencias digitales de los estudiantes y docentes de los programas participantes al entorno educativo ac- tual, permitirá corroborar la pertinencia de los saberes de los actores del proceso educativo a las necesidades actuales del contexto. Así mismo resultará conveniente.

\section{Material y método}

\section{Participantes}

La muestra corresponde a 1366 registros, de igual número de estudiantes del área de la educación física y el deporte, de tres instituciones de educación superior de la ciudad de Bogotá-Colombia, de los cuales 1086 corresponden al género masculino y 280 al femenino.

Por su parte, la muestra de docentes corresponde a un total de 104 participantes (78 hombres y 26 mujeres) de las mismas instituciones.

\section{Instrumentos}

Para medir la autopercepción de competencias digitales en los estudiantes, se empleó el cuestionario para el estudio de la Competencia Digital del Alumnado de Educación Superior (CDAES), propuesto por Gutiérrez-Castillo, Cabero-Almenara y Estrada-Vidal (2017). El instrumento se compone de seis dimensiones (alfabetización tecnológica; búsqueda y tratamiento de la información; pensamiento crítico, solución de problemas y toma de decisiones; comunicación y colaboración; ciudadanía digital; y creatividad e innovación), que agrupan 22 indicadores, evaluados a través de una escala de Likert (ajustada para el presente estudio a cinco opciones de respuesta) para 43 afirmaciones, en la cual el valor uno hace referencia a que el estudiante se siente completamente incompetente para realizar la actividad planteada y cinco, la dominación completa de la declaración. La consistencia interna del cuestionario, medida con el coeficiente alfa de Cronbach, arroja una calificación de .96, lo que asegura su fiabilidad y validez. Los datos resultantes se clasificaron en cinco intervalos a partir de su valor medio así: $\bar{x}<1.8=$ percepción baja; $1.8<<2.6=$ percepción media baja; $2.6<<3.4=$ percepción media; $3.4<<4.2=$ percepción media alta; $>4.2=$ percepción alta.

Para la medición de la percepción de los docentes, se utilizó el cuestionario sobre competencia digital propuesto por Guizado, Menacho y Salvatierra (2019). Este instrumento, que consta de 25 ítems distribuidos en cinco dimensiones: aprendizaje, informacional, comunicativa, cultura digital y tecnológica, reportó una confiabilidad de .77 en el coeficiente alfa de Cronbach y de .75 en la aplicación del método Delphi. Los datos fueron evalua- 
dos a través de una escala de Likert con cuatro opciones de respuesta, siguiendo la metodología propuesta por los autores. Los resultados se clasificaron en cinco intervalos a partir de su valor medio así: $<1.6=$ percepción baja; $1.6<<2.2=$ percepción media baja; $2.2<$ $<2.8=$ percepción media; $2.8<<3.4=$ percepción media alta; $>4=$ percepción alta.

\section{Procedimiento}

Para este estudio de tipo cuantitativo descriptivo, previa aprobación del Comité de Ética, se procedió a la toma de datos a través de la aplicación Google Formulario para estudiantes y docentes de los cuatro programas participantes. Este proceso se realizó durante el segundo semestre del año 2020.

\section{Análisis de datos}

Para el análisis estadístico de la información se fijó un nivel de significancia de .05, con un 95\% de confianza. Para la evaluación de la normalidad de los datos se empleó la prueba de Kolmogorov Smirnov. Para las pruebas de diferencia de medias se utilizó la prueba no paramétrica de Kruskal Wallis básica y post-hoc. Para el análisis de la correlación entre variables se empleó el coeficiente de Spearman, en consideración con la naturaleza de las variables bajo estudio. Todos los procedimientos se realizaron con el programa estadístico SPSS v. 25 .

\section{Resultados}

De los 1366 estudiantes participantes, el 79.5\% corresponde a hombres (1086) y el 20.5\% (280) a mujeres (Tabla 1).

Los programas profesionales en Colombia se encuentran organizados por el sistema de créditos académicos, con duración entre ocho y diez semestres. Tal como se aprecia en la tabla 1, los estudiantes de la muestra provienen de todos los periodos académicos de formación.

En conjunto, las tres instituciones participantes albergan un total de 2375 estudiantes, en cuatro diferentes programas de formación profesional del área de la educación física y el deporte, por cuanto la muestra corresponde al 57.5\% del total de estudiantes inscritos en estos cuatro programas, para el segundo semestre del año 2020.

Los programas, que en adelante serán referidos como P1, P2, P3 y P4, tal como se presenta en la tabla 2, responden a la oferta educativa del nivel profesional en las modalidades distancia y presencial (Tabla 2).
Así mismo, conviene señalar que los programas P1 y P2 otorgan títulos de licenciatura, lo cual perfila a sus egresados hacia el ejercicio de la docencia, por cuanto su plan de estudios incluye asignaturas del componente pedagógico, mientras que los programas P3 y P4 ofrecen una formación con mayor énfasis en las ciencias del deporte.

Por su parte, la muestra de docentes corresponde a un total de 104 participantes de los cuatro programas de formación, tal como se presenta en la tabla 3. De ellos, el 38\% (40) cuentan con maestría como máximo nivel de formación, el 37\% (38) no tiene formación posgradual, el 23\% (24) es especialista, y tan solo el 2\% ostentan el título de doctorado. En relación con la experiencia profesional, el grupo registra un promedio de 6.5 años, con una mayor concentración en el intervalo de uno a cinco años (Tabla 3).

Respecto de los resultados generales del CDAES, la tabla 4 consolida la concentración relativa de la muestra en los diferentes niveles de percepción. Tal como se puede apreciar, los estudiantes consideran, mayoritariamente, que sus competencias digitales se encuentran en un nivel medio alto (Tabla 4).

Una vez abordados los descriptivos generales de la muestra, se procedió a evaluar la normalidad de los datos a través de la prueba Kolmogorov-Smirnov. Con una significancia del .05, tanto los datos del cuestionario de estudiantes como de docentes, indican un $p$ value $=.000^{\mathrm{c}}$, señalando que los resultados no siguen una distribución normal. Con sustento en lo anterior, en

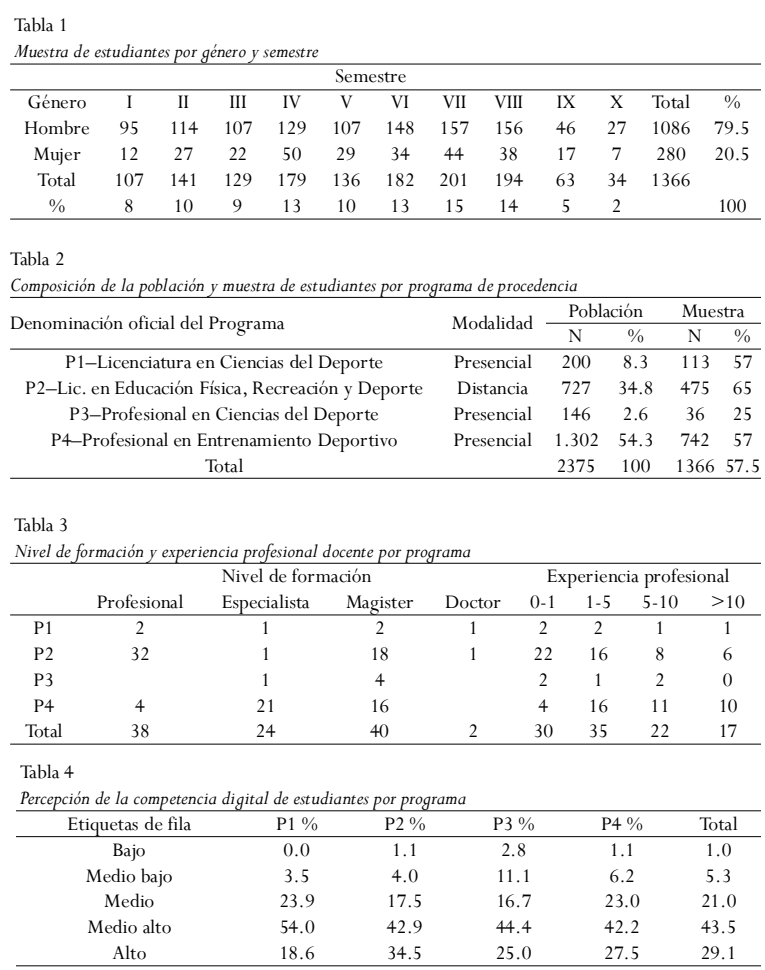


adelante se realizarán pruebas estadísticas no paramétricas.

Se presentan a continuación los resultados del cuestionario aplicado a estudiantes. Para determinar la existencia de diferencias en la percepción promedio por cada una de las dimensiones del CDAES, en función de su género, se realizó la prueba de Kruskal Wallis para diferencia de medianas. Con una significancia de .05, los resultados del contraste no permiten rechazar la hipótesis de igualdad (Tabla 5).

El mismo procedimiento estadístico se aplicó para los datos por dimensión evaluada, ahora en función del programa. Con una significancia del .05, los resultados de la tabla 6 revelan que existen diferencias significativas en al menos uno de los programas comparados para todas las dimensiones que componen el CDAES (Tabla 6).

Para conocer cuáles son las muestras que presentan diferencias y generan el rechazo de la hipótesis de igualdad, se realiza la prueba de Kruskal Wallis post hoc para comparaciones múltiples. Los resultados se presentan para cada una de las dimensiones del CDAES (Tabla 7).

\begin{tabular}{|c|c|c|c|c|c|c|c|}
\hline \multicolumn{8}{|c|}{ Dimensión1 Dimensión2 Dimensión3 Dimensión4 Dimensión5 Dimensión6 } \\
\hline \multirow{2}{*}{$\begin{array}{c}\text { Kruskal Wallis } \\
P\end{array}$} & 1.860 & .694 & .285 & .009 & \multicolumn{2}{|c|}{.097} & .105 \\
\hline & .173 & .405 & .594 & 924 & .7 & & .746 \\
\hline \multicolumn{8}{|l|}{ Tabla 6} \\
\hline \multicolumn{8}{|c|}{ Resultados del CDAES en función del programa de procedencia de los estudiantes } \\
\hline & $\begin{array}{c}\text { Dimensión } \\
1\end{array}$ & Dimensión2 & $\begin{array}{c}\text { Dimensión } \\
3\end{array}$ & $\begin{array}{c}\text { Dimensión } \\
4\end{array}$ & \multicolumn{2}{|c|}{ Dimensión5 } & $\begin{array}{c}\text { Dimensión } \\
6\end{array}$ \\
\hline $\begin{array}{l}\text { Kruskal Wallis } \\
\text { S }\end{array}$ & 10.471 & 16.448 & 31.524 & 21.287 & 38. & & 30.484 \\
\hline & 015 & .001 & .000 & .000 & & & .000 \\
\hline \multicolumn{8}{|l|}{ Tabla 7} \\
\hline \multirow{2}{*}{\multicolumn{8}{|c|}{$\frac{\text { Prueba de Kruskal Wallis post hoc - Dimensiones }}{\text { Mediana }}$}} \\
\hline & Median & & tico & \multicolumn{2}{|c|}{ Media } & \multicolumn{2}{|c|}{ Mediana } \\
\hline \multirow{6}{*}{ Dimensión 1} & P1-P2 & 72.606 & .078 & 3.887 & 3.959 & 3.923 & 4.000 \\
\hline & P1-P3 & 51.380 & .496 & 63.887 & 3.709 & 3.923 & 3.807 \\
\hline & P1-P4 & -5.736 & .885 & 3.887 & 3.848 & 3.923 & 3.923 \\
\hline & P2-P3 & 123.98 & .069 & 9 3.959 & 3.709 & 4.000 & 3.807 \\
\hline & P2-P4 & 66.870 & .004 & $+\quad 3.959$ & 3.848 & 4.000 & 3.923 \\
\hline & P3-P4 & -57.11 & .396 & $6 \quad 3.709$ & 3.848 & 3.807 & 3.923 \\
\hline \multirow{6}{*}{ Dimensión 2} & P1-P2 & -85.43 & .038 & 3.802 & 3.915 & 3.833 & 4.000 \\
\hline & P1-P3 & 32.172 & .669 & 9 3.802 & 3.625 & 3.833 & 3.916 \\
\hline & P1-P4 & 3.945 & .921 & 13.802 & 3.753 & 3.833 & 3.833 \\
\hline & P2-P3 & 117.60 & .083 & $3 \quad 3.915$ & 3.625 & 4.000 & 3.916 \\
\hline & P2-P4 & 89.378 & .000 & 3.915 & 3.753 & 4.000 & 3.833 \\
\hline & P3-P4 & -28.22 & .674 & $\begin{array}{l}+\quad 3.625 \\
\end{array}$ & 3.753 & 3.916 & 3.833 \\
\hline \multirow{6}{*}{ Dimensión 3} & P1-P2 & -137.25 & .001 & 13.590 & 3.855 & 3.750 & 4.000 \\
\hline & P1-P3 & 34.838 & .642 & 3.590 & 3.479 & 3.750 & 3.625 \\
\hline & P1-P4 & -18.21 & .646 & $6 \quad 3.590$ & 3.603 & 3.750 & 3.750 \\
\hline & P2-P3 & 172.09 & .011 & 13.855 & 3.479 & 4.000 & 3.625 \\
\hline & P2-P4 & 119.04 & .000 & 3.855 & 3.603 & 4.000 & 3.750 \\
\hline & P3-P4 & -53.05 & .428 & 3.479 & 3.603 & 3.625 & 3.750 \\
\hline \multirow{6}{*}{ Dimensión 4} & P1-P2 & -113.26 & .006 & 53.663 & 3.843 & 3.666 & 4.000 \\
\hline & P1-P3 & 53.217 & .480 & 3.663 & 3.490 & 3.666 & 3.666 \\
\hline & P1-P4 & -18.40 & .644 & +3.663 & 3.668 & 3.666 & 3.777 \\
\hline & P2-P3 & 166.47 & .014 & $4 \quad 3.843$ & 3.490 & 4.000 & 3.666 \\
\hline & P2-P4 & 94.859 & .000 & 3.843 & 3.668 & 4.000 & 3.777 \\
\hline & P3-P4 & -71.61 & .287 & $7 \quad 3.490$ & 3.668 & 3.666 & 3.777 \\
\hline & P1-P2 & -189.68 & .000 & 3.663 & 3.980 & 3.666 & 4.000 \\
\hline & P1-P3 & -19.90 & .791 & 13.663 & 3.615 & 3.666 & 3.833 \\
\hline Dimensión 5 & P1-P4 & -68.45 & .084 & +3.663 & 3.668 & 3.666 & 3.833 \\
\hline & P2-P3 & 169.78 & .012 & $2 \quad 3.980$ & 3.615 & 4.000 & 3.833 \\
\hline & P2-P4 & 121.23 & .000 & 3.980 & 3.668 & 4.000 & 3.833 \\
\hline & P3-P4 & -48.55 & .469 & 3.615 & 3.668 & 3.833 & 3.833 \\
\hline & P1-P2 & -156.22 & .000 & 3.647 & 3.915 & 3.800 & 4.000 \\
\hline & P1-P3 & 28.669 & .703 & $\begin{array}{l}3 \quad 3.647 \\
\end{array}$ & 3.477 & 3.800 & 3.600 \\
\hline Dimensión 6 & P1-P4 & -47.48 & .231 & 13.647 & 3.705 & 3.800 & 3.800 \\
\hline & P2-P3 & 184.89 & .006 & $6 \quad 3.915$ & 3.477 & 4.000 & 3.600 \\
\hline & P2-P4 & 108.74 & .000 & 3.915 & 3.705 & 4.000 & 3.800 \\
\hline & P3-P4 & -76.15 & .255 & 3.477 & 3.705 & 3.600 & 3.800 \\
\hline
\end{tabular}

La comparación entre parejas de la tabla 7 permite afirmar que existen diferencias significativas entre la percepción de los estudiantes de los programas P2 y P4, en relación con las competencias abordadas en la dimensión de alfabetización tecnológica, siendo mejor para los primeros.

Para el caso de la dimensión de búsqueda y tratamiento de la información se ratifican diferencias entre los programas $\mathrm{P} 2$ y $\mathrm{P} 4$, y se evidencian también entre los programas P1 y P2. En ambos casos, la percepción de los estudiantes del programa P2 frente a las competencias propias de esta dimensión es ligeramente superior.

Sobre la dimensión de pensamiento, solución de problemas y toma de decisiones, en ella se aprecian diferencias estadísticamente relevantes del programa P2 frente a los programas P1, P3 y P4.

Los hallazgos se reproducen para el análisis de las dimensiones de comunicación y colaboración y ciudadanía digital; el programa P2 muestra medidas de tendencia central estadísticamente diferentes a los programas P1, P3 y P4. Tanto en la dimensión cuatro como en la dimensión cinco, la percepción de los estudiantes del programa P2 sobre sus competencias tecnológicas es superior al de sus pares de los programas P1, P3 y P4.

En la dimensión de creatividad e innovación, los resultados de la prueba post hoc de Kruskal Wallis son similares a los evidenciados previamente; tan solo las percepciones de los estudiantes del programa P2 se muestran diferentes.

También se calculó el coeficiente de correlación de Spearman para los datos de percepción por dimensión frente a la edad y el semestre cursado por el participante. Los resultados de la tabla 8 , señalan que con un nivel de significancia del .05 la edad tiene una correlación positiva con la percepción de competencias digitales, no obstante, el coeficiente de rho es tan pequeño en todos los casos que apenas sugiere una correlación lineal débil. Una situación similar se presenta con la variable semestre cuyo estadístico de contraste sugiere correlación excepto con las dimensiones cinco y seis, pero su coeficiente rho es demasiado pequeño para ser considerado relevante (Tabla 8 ).

Los resultados del cuestionario realizado a los docentes se presentan en la tabla 9. La distribución de frecuencias global y por dimensión indica que, mayoritariamente, los docentes evalúan el desarrollo de sus competencias digitales en un nivel medio alto y alto (Tabla 9).

El resultado de la prueba de Kruskal Wallis para el 
puntaje global por participante en función del programa al que pertenecen arroja un $p$-value de .648 por cuanto no se rechaza la hipótesis nula. Lo anterior se replica para el análisis del puntaje por dimensión en función del programa con $p$-value de .233; .449; .637; .563;.477 para las dimensiones uno a cinco, respectivamente; tampoco se presentan diferencias por género.

Contrariamente los resultados para el puntaje global por participante en función de la formación arrojan un $p$-value de .036, lo que nos lleva a hacer el análisis post hoc; los resultados se presentan en la tabla 10. Tal como se aprecia hay diferencias estadísticamente significativas entre los resultados de los docentes con formación posgradual y aquellos que no la tienen (Tabla 10).

Finalmente se obtuvo el coeficiente de correlación de Spearman para el puntaje global de la prueba frente a la experiencia, que con un $p$-value $=.000 \mathrm{y}$ un $r h o=$ .344 señala una correlación media entre las dos variables. En la tabla 11 se presentan los resultados de la misma prueba, ahora por dimensión evaluada, en todos los casos existe correlación media (Tabla 11).

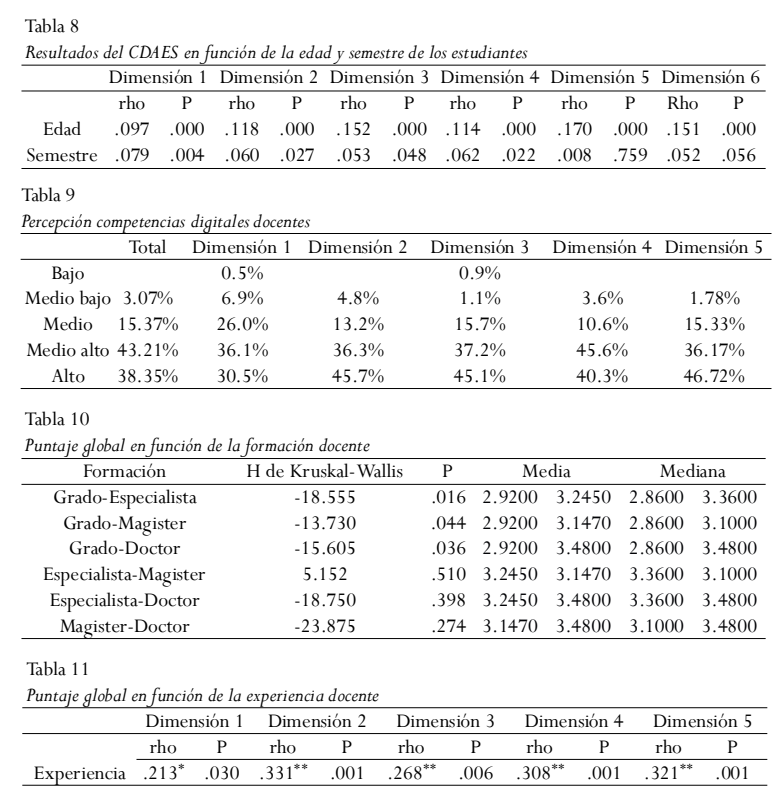

\section{Discusión}

La composición de la muestra ratifica la masculinización en la formación profesional de la actividad física y el deporte en todos los niveles, evidenciada en otros estudios (Serra, 2016; Serra, Soler, Vilanova, \& Hinojosa-Alcalde, 2019). Por su parte, los resultados niegan diferencias estadísticamente significativas en la percepción general de los estudiantes sobre sus competencias digitales, en función del género, en la misma línea de lo hallado por Díaz-Barahona, Molina-García y
Monfort-Pañego (2019) y Guillén-Gámez y Perrino (2020). Este es un hecho relevante pues desvirtúa en la población, la presencia del concepto de segunda brecha digital, que refleja las diferencias entre mujeres y hombres respecto a los usos y las habilidades en TIC y que podría constituirse en barrera para la efectiva incorporación de las mujeres a la sociedad de la información (Castaño, 2008).

Tampoco se revela una correlación importante entre la edad de los participantes y su dominio de las TIC, contrario a lo encontrado por (Martínez \& RodríguezGarcía, 2018), quien reporta que la brecha digital aumenta a medida que avanza la edad.

Tal como señalan Cabezas, Casillas, Sánchez-Ferreira y Teixeira (2017), la influencia de las variables género y edad en la adquisición de la competencia digital en estudiantes ha sido objeto de estudio de distintas investigaciones, y los hallazgos son disímiles entre sí. Para el caso de la edad lo más común es encontrar resultados que indican diferencias a favor de los más jóvenes e incluso algunos señalan el aumento de problemas de uso de las TIC con la edad, sin embargo, un número considerable, como en nuestro caso, reporta no haber encontrado diferencias.

Para este caso, en un ambiente particularmente juvenil y masculino, no hay evidencia estadística que permita establecer que estas variables tienen efectos relevantes sobre el desarrollo de las competencias digitales de los estudiantes.

Ahora bien, la puntuación promedio de la muestra en el CDAES, indica que los estudiantes del área de la educación física y el deporte perciben el desempeño de sus competencias digitales en un nivel medio alto, con una media global de 3.80. Ratificando lo anterior, la distribución de frecuencias de la tabla 5 , indica que el $43.5 \%$ de los estudiantes, reporta una puntuación media alta para el cuestionario, seguida por el $29.1 \%$, que registra una puntuación alta. Este es un hallazgo relevante, pues se presenta en contravía con lo hallado en otras investigaciones realizadas con estudiantes del área, cuyos resultados fueron medios y medios-bajos (Cabrera, 2020; Pino \& Soto, 2010; Romero-Martín, CastejónOliva, López-Pastor, \& Fraile-Aranda, 2017).

Las puntuaciones promedio más altas por dimensión se ubican en el componente de alfabetización, que hace referencia a la manera en la cual los estudiantes entienden y usan las TIC, seleccionan y emplean aplicaciones y transfieren el conocimiento existente para el aprendizaje de nuevas tecnologías, mientras que la percepción más baja corresponde a la dimensión de solución de pro- 
blemas y toma de decisiones, competencias fundamentales para el ejercicio profesional; estos resultados concuerdan con los de Conde, Trujillo y Castaño (2017). De acuerdo con lo anterior, la mayor parte de los estudiantes se considera capaz de utilizar de manera eficiente, navegadores, el correo electrónico, servicios de mensajería instantánea y aplicaciones en general, pero no se perciben suficientemente capacitados para configurar y resolver problemas personales, sociales y profesionales reales, a partir del uso de estas y otras herramientas digitales. A priori, estos resultados pueden parecer naturales teniendo en cuenta que se trata de profesionales en formación que aún no terminan de cursar su plan curricular, y por tanto estas competencias pueden encontrarse aún en desarrollo (Esteve-Mon, GisbertCervera, \& Lázaro-Cantabrana, 2016). Lo anterior pareciera encontrar sustento estadístico al comprobar que el puntaje medio para esta dimensión mejora a medida que se sustraen los estudiantes de los semestres más bajos, sin embargo, el coeficiente de correlación rho por semestre cursado indica que la relación lineal entre estas variables es débil.

De otra parte, los resultados de las pruebas básicas y post hoc de Kruskall Wallis, permiten establecer diferencias estadísticas entre los resultados obtenidos por los estudiantes del programa de modalidad distancia (P2), frente a sus similares de la modalidad presencial, en todas las dimensiones evaluadas. Una explicación inicial reposa en la inclusión de un mayor porcentaje de créditos académicos tendientes al fortalecimiento de las competencias digitales en la malla curricular de este programa y en la exposición habitual a la tecnología como medio facilitador del aprendizaje, pues los entornos virtuales de aprendizaje ofrecen un gran abanico de recursos y facilidades que potencian las competencias de aprender a aprender y la autonomía e iniciativa personales de docentes y estudiantes (Prat Ambrós, Camerino Foguet, \& Coiduras Rodríguez, 2013; Díaz Barahona, Molina-García, \& Monfort-Pañego, 2020).

No obstante, al evaluar este factor de manera global en la muestra, se evidencia que el programa P1 (que no incluye ninguna asignatura de tal naturaleza) le sigue al P2 en percepción de desempeño digital, por encima de otros que sí contemplan estos escenarios académicos. Lo que nos lleva a pensar en la posibilidad de que sea su orientación pedagógica el elemento diferenciador, pues en ambos casos se trata de programas de formación docente.

Las TIC y su creciente injerencia en todos los escenarios sociales, han representado una transformación de la cultura pedagógica, pues el conocimiento ha pasado a ser un bien social de carácter universal, por su accesibilidad y pluralidad, que mediado por recursos digitales adecuados puede potenciar la experiencia educativa y motivar el proceso de enseñanza-aprendizaje (Aguirre \& Ruiz, 2012). Este abordaje conceptual desde otras perspectivas o escenarios académicos, no estrictamente vinculados a las competencias digitales, puede promover un mayor interés y por tanto un mejor desempeño de los estudiantes con vocación docente, frente a aquellos con enfoque más disciplinar, lo que pone de manifiesto que las competencias digitales pueden ser desarrolladas de manera transversal a lo largo de la formación de los estudiantes.

Un segundo elemento explicativo puede ser la constante utilización de recursos tecnológicos de formación asincrónica, además de las herramientas para administrar la actividad del estudiante, a las que estos se ven expuestos en el marco de la modalidad educativa a distancia, contra la concentración y presencialidad propia de los entornos tradicionales (Barrios, Domínguez, \& Barreto, 2018). Este hecho podría sugerir que los docentes de tales programas cuentan con un conjunto superior de conocimientos y habilidades para utilizar las herramientas tecnológicas como recursos educativos integrados en su práctica diaria, no obstante, los resultados estadísticos muestran que no existen diferencias entre la percepción de los docentes en función del programa al que pertenecen.

En concordancia con los resultados de los estudiantes, los docentes perciben el desarrollo de sus competencias digitales en un nivel medio alto, lo cual resulta coherente y esperanzador, toda vez que un docente que no se sienta competente no podrá lograr el desarrollo de estas competencias en sus estudiantes. Los hechos en comento podrían contrastar la brecha digital entre estudiantes «nativos digitales» y profesores «inmigrantes digitales» evidenciada en otros estudios (Fernández-Cruz \& Fernández-Díaz, 2016). Los mejores resultados se dan para docentes con formación posgradual, lo que pone de manifiesto la necesidad de la continua formación del cuerpo docente, con el objeto de dotarlo de las herramientas requeridas en el entorno educativo contemporáneo. Por último, no menos relevante, resulta la correlación positiva existente entre la experiencia docente y su percepción en competencias TIC. Los resultados de esta investigación sugieren que el dominio de los diferentes entornos de aprendizaje permite la generación de escenarios transformadores y significativos en el ámbito académico (Huber, 2008), dotando a profeso- 
res de nuevas herramientas y destrezas para el desarrollo de su quehacer, lo que les permite autopercibirse más competentes en la utilización de las herramientas digitales en el aula.

\section{Conclusiones}

Los resultados de esta investigación indican que no se presentan diferencias significativas en la percepción del desarrollo de las competencias digitales en función del género, ni la edad, de estudiantes y docentes del área de la actividad física y el deporte. Tanto estudiantes como docentes del área de la educación física y el deporte se autoperciben competentes en un nivel medio alto en el manejo y utilización de las TIC. Uno de los hallazgos más relevantes del presente estudio es que la percepción de competencia digital de los estudiantes de la modalidad distancia es superior a la de sus homólogos de la modalidad presencial, lo cual podría explicarse por la continua exposición de los primeros a herramientas TIC, tanto para el desarrollo del plan curricular como para gestionar los diferentes espacios estudiantiles.

Así mismo, las pruebas estadísticas indican que los estudiantes del área de la actividad física y el deporte con enfoque de formación docente se consideran más competentes para utilizar las herramientas tecnológicas, que aquellos con enfoque disciplinar, lo cual podría atribuirse a la utilización de las TIC desde los ámbitos didáctico y pedagógico. Este hecho pondría de manifiesto la eficacia del desarrollo de competencias digitales transversalmente, desde asignaturas aparentemente ajenas al entorno tecnológico.

Los estudiantes sugieren que el área de mayor dominio, es la relacionada con la utilización de herramientas TIC básicas, como el correo electrónico, aulas virtuales y apps en general, sin embargo, se sienten menos competentes para emplear las herramientas digitales en el análisis y resolución de problemas reales, lo cual se presenta como señal de alarma ante la posibilidad de que las TIC estén siendo abordadas como un mero instrumento de búsqueda y transferencia de información, y no como vehículo transformador del proceso de enseñanza-aprendizaje, en el marco de la sociedad del conocimiento.

El nivel de formación y la experiencia de los docentes, por su parte, tienen un impacto significativo en el desarrollo de sus propias competencias digitales, a mayor nivel de formación y/o años de experiencia docente, mejor la percepción de estos frente a su desempeño. Este hecho es una manifestación fáctica de la necesidad de la formación continua del cuerpo profesoral de los programas de educación superior, y se vislumbra como herramienta para cerrar la brecha digital existente entre estos y los estudiantes, considerados per se competentes.

Estos hallazgos alimentan el cuerpo académico de investigación en un área cada vez más relevante de la educación universitaria. Para futuras investigaciones, resulta deseable incluir también una prueba objetiva de valoración de competencias digitales, que permita establecer si la percepción de estudiantes y docentes se encuentra sobrevalorada, pues diferentes estudios internacionales sugieren que el nivel global es muy inferior al encontrado en este y otros estudios similares. Así como una investigación de tipo cualitativo, que permita contrastar sus resultados con los arrojados por investigaciones de índole más cuantitativa. Por último, sería interesante plantear un diseño de investigación en el que se correlacionen los resultados de los estudiantes con los docentes uno a uno, podría servir para establecer la influencia que los maestros generan sobre sus discípulos en el desarrollo de estas competencias cada vez más necesarias y reclamadas por la sociedad contemporánea.

\section{Referencias}

Aguirre, G., \& Ruiz, Ma. del R. (2012). Competencias digitales y docencia: una experiencia desde la práctica universitaria. Innovación Educativa, 12(59), 121-141.

Araújo, J. G. E., Batista, C., \& Moura, D. L. (2017). Exergames in physical education: A systematic review. Movimento, 23(2), 529542.https://doi.org/10.22456/1982-8918.65330

Barrios, J.C., Domínguez,A., \& Barreto, C. R. (2018). Fortalecimiento de la competencia TIC de estudiantes de educación superior en AmbientesVirtuales de Aprendizaje. Revista Espacios, 39(25), 35 undefined. Retrieved from https: / / www.revistaespacios.com/ a18v39n25/a18v39n25p35.pdf

Batane,T., \& Ngwako,A. (2017).Technology use by pre-service teachers during teaching practice:Are new teachers embracing technology right away in their first teaching experience? Australasian Journal of Educational Technology, 33(1), 48-61. Retrieved from https:// doi.org/10.14742/ajet.2299

Cabero-Almenara, J., \& Palacios-Rodríguez,A. (2019). Marco Europeo de Competencia Digital Docente «DigCompEdu». Traducción y adaptación del cuestionario «DigCompEdu Check-In». Revista de Educación Mediática yTIC, 9(1), 213-234.https://doi.org/ 10.21071/edmetic.v9i1.12462

Cabezas, M., Casillas, S., Sanches-Ferreira, M., \& Teixeira, F. L. (2017). ¿Condicionan el género y la edad el nivel de competencia digital? Un estudio con estudiantes universitarios. Fonseca, Journal of Communication, 15, 109-125. https://doi.org/10.14201/ fjc201715109125

Cabrera,J.F. (2020). Producción científica sobreintegración deTIC a la 
Educación Física. Estudio bibliométrico en el periodo 1995-2017. Retos, 37, 748-754. Retrieved from www.retos.org

Castaño, C. (2008). La segunda Brecha Digital. Madrid: Cátedra.

Cela-Ranilla,J.M.,Esteve,V., Esteve, F., González,J., \& Gisbert-Cervera, M. (2017). El docente en la sociedad digital: unapropuesta basada en la pedagogía transformativa y en la tecnología avanzada. Currículum y Formación de Profesorado, 21(1), 403-422.

Conde, E., Trujillo, J.J., \& Castaño, H. (2017). Descifrando el currículum a través de las TIC: una visión interactiva sobre las competencias digitales de los estudiantes de Ciencias del Deporte y de la Actividad Física. Revista de Humanidades, 31, 195-214.

Díaz-Barahona, J., Molina-García, J., \& Monfort-Pañego, M. (2019). Estudio de las actitudes y el interés de los docentes de primaria de educación física por las TIC en la Comunidad Valenciana. Retos: Nuevas Tendencias En Educación Física, Deportey Recreación, 35, 267 272. Retrieved from www.retos.org

Díaz Barahona,J., Molina-García, J., \& Monfort-Pañego, M. (2020). El conocimiento y la intencionalidad didáctica en el uso deTIC del profesorado de educación física. Retos: Nuevas Tendencias En Educación Física, Deporte y Recreación, 38, 497-504. Retrieved from www.retos.org

Domingo-Coscollola,M., Onsès-Segarra,J., \& Sancho-Gil,J.M.(2018). La cultura DIY en educación primaria.Aprendizaje transdisciplinar, colaborativo y compartido en DIYLabHub. Revista de Investigacion Educativa, 36(2), 491-508. https://doi.org/10.6018/ rie.36.2.304421

Esteve-Mon, F. M., Gisbert-Cervera, M., \& Lázaro-Cantabrana, J. L. (2016). La competencia digital de los futuros docentes: ¿Cómo se ven los actuales estudiantes de educación? Perspectiva Educacional, 55(2), 38-54.https://doi.org/10.4151/07189729-vol.55-iss.2art. 412

Fernández, E., Ordóñez, E., Morales, B., \& López, J. (2019). La competencia digital en la docencia universitaria (Primera edición). Barcelona: Octaedro.

Fernández-Cruz, F.J., \& Fernández-Díaz, M. J. (2016). Los docentes de la Generación Z y sus competencias digitales. Comunicar, 46(26), 95-105.https: / /doi.org/10.3916/C46-2016-10

Flores-Lueg, C., \& Roig, R. (2016). Diseño y validación de una escala de autoevaluación de competencias digitales para estudiantes de pedagogía. Revista de Medios y Educación, 48, 209-224. https://doi.org/ 10.12795/pixelbit.2016.i48.14

González-Zamar, M.-D., Abad-Segura, E., \& Belmonte-Ureña, L. J. (2020).Aprendizaje significativo en el desarrollo de competencias digitales. Análisis de tendencias. IJERI: International Journal of Educational Research and Innovation, 14, 91-110. https: / /doi.org/ 10.46661/ijeri.4741

Guillén-Gámez,F. D. \& \& Perrino, M. (2020).Análisis Univariante de la Competencia Digital en Educación Física un estudio empírico. Retos: Nuevas Tendencias En Educación Física, Deporte y Recreación, 37, 326-332. Retrieved from www.retos.org

Guizado, F., Menacho, I., \& Salvatierra,A. (2019). Competencia digital y desarrollo profesional de los docentes de dos instituciones de educación básica regular del distrito de Los Olivos, Lima-Perú. HAMUT'AY, 6(1), 54-70. https://doi.org/10.21503/ hamu.v6i1.1574

Gutiérrez-Castillo, J.-J., Cabero-Almenara, J., \& Estrada-Vidal, L.-I. (2017). Diseño y validación de un instrumento de evaluación de la competencia digital del estudiante universitario. Revista ESPACIOS,
38(10), 16 43. Retrievedfrom http:/ / www.revistaespacios.com/ a17v38n10/17381018.html

Guzmán-Simón, F., García-Jiménez, E., \& López-Cobo, I. (2017). Undergraduate students' perspectives on digital competence and academic literacy in a Spanish University. Computers in Human Behavior, 74,196-204.https://doi.org/10.1016/j.chb.2017.04.040 Huber, G. L. (2008). Aprendizaje activo y metodologías educativas Active learning and methods of teaching. Revista de Educacion, 59-81.

Llorente, M. del C., \& Cabero, J. (2010). Desarrollo de un instrumento sobre competencias TIC en alumnos universitarios. Congreso EuroIberoamericano de Alfabetización Mediática y Culturas Digitales.

López-Gil, K. S., \& Sevillano, M. L. (2020). Desarrollode competencias digitales de estudiantes universitarios en contextos informales de aprendizaje. Educatio Siglo XXI, 38(1), 53-78. https: / /doi.org/ 10.6018/educatio.413141

Marcano, B., Íñigo,V., \& Sánchez,J.M. (2020).Validación de rúbricapara evaluación dee-actividades diseñadas para el logro de competencias digitales docentes. Apuntes Universitarios, 10(2), 115-129. https: / / doi.org/10.17162/au.v10i2.451

Martínez,N., \& Rodríguez-García,A.-M. (2018).Alfabetización y competencia digital en personas mayores: el caso del aula permanente deformación abierta de la Universidad de Granada (España). RevistaESPACIOS, 39(10), 37.

Merino-Campos, C., \& del Castillo, H. (2016). Los beneficios de los videojuegos interactivos: una aproximación educativa y una revisión sistemática de la actividad física. Journal of New Approaches in Educational Research, 5(2), 115-122. https://doi.org/10.7821/ naer.2016.7.164

Pino, M. \& Soto, J. (2010). Identificación del dominio de competencias digitales en el alumnado del grado de magisterio. Teoría de La Educación. Educación y Culturaen La Sociedad de La Información, 11(3), 336 362. Retrieved from https://www.redalyc.org/pdf/2010/ 201021093015.pdf

Prat Ambrós, Q., Camerino Foguet, O., \& Coiduras Rodríguez, J. L. (2013). Introducción de lasTIC eneducación física. Estudio descriptivo sobre la situación actual. Apunts Educación Física y Deportes, 113(3), 37-44. https://doi.org/http://dx.doi.org/10.5672/ apunts.2014-0983.es.(2013/3).113.03

Quijada, R. (2015).Tecnologías de la Información y la Comunicación (TIC) en Educación Física. Sportis Scientific Technical Journal , 1(1), 75-86. Retrieved from www.sportis.es

Rodríguez-García,A. M., Raso, F., \& Ruiz-Palmero, J. (2019). Competencia digital, educación superior y formación del profesorado: un estudio de meta-análisis en laWeb of Science. Pixel-Bit, Revista de Medios y Educacion, 54, 65-81. https://doi.org/10.12795/ pixelbit.2019.i54.04

Romero-Martín, M. R., Castejón-Oliva, F. J., López-Pastor,V. M., \& Fraile-Aranda, A. (2017). Evaluación formativa, competencias comunicativas yTIC en la formación del profesorado. Comunicar, 25(52), 73 -82.https://doi.org/10.3916/C52-2017-07

Serra, P. (2016). La perspectiva de género en los estudios de ciencias de la actividad fisica y el deporte. Barcelona. Retrieved from https:// www.tdx.cat/bitstream/handle/10803/402513/ PSP_TESIS.pdf?sequence $=1$

Serra, P., Soler, S., Vilanova, A., \& Hinojosa-Alcalde, I. (2019). Masculinización en estudios de las ciencias de la actividad física y el deporte. Apunts Educación Fisica y Deportes, 135(1), 9-25.https: / / doi.org/10.5672/apunts.2014-0983.es.(2019/1).135.01 